\title{
FIXATION AND RECALL OF YOUTUBE AD BANNERS: AN EYE-TRACKING STUDY
}

\author{
Chatpong Tangmanee \\ Chulalongkorn University \\ Chulalongkorn Business School, Chulalongkorn University, Bangkok, \\ Thailand, 10330 \\ chatpong@cbs.chula.ac.th
}

\begin{abstract}
Attaching an ad banner on a clip in a video-sharing website such as YouTube has become common although eye-tracking studies have concluded that this fails to secure visitors' attention. To date, there have been no studies verifying whether ad banners on a video clip can ensure eye fixation from viewers. Through eye-tracking, this study investigates whether YouTube visitors fixate on ad banners, what the correlations between fixation duration on banners and overall fixation counts are, and the extent to which site visitors are able to recall details of ad banners and of the clip viewed. Using a Miramatrix eye-tracker to record YouTube viewers' eye movements, this study showed that nearly all fixated at least once on an ad banner in a clip. However, less than $10 \%$ were able to correctly recall the ad content viewed. Nevertheless, about half of viewers were able to correctly recall clip details. Fixation duration on the banner and fixation counts on the clip are negatively correlated, but the relationship between fixation duration and counts on the banner was insignificant. This study sheds new light on YouTube advertising through the use of eye-tracking and advises advertisers to be attentive in selecting clips on which ad banners will appear.
\end{abstract}

Keywords: Fixation, Recall, Eye-tracking, Ad Banner, YouTube

\section{PROBLEM STATEMENT}

According to a fair amount of empirical evidence, Internet banners may not be so useful for marketing promotion as was thought decades $\operatorname{ago}^{1,2}$. Hervet et al. ${ }^{3}$ confirm that Internet users avoid looking at banners. This effect was not incidental, but was due to viewers' direct intent to refrain from looking at banners. Moreover, viewers showed poor recall and 
recognition of content that appeared on banners; however, these studies were mostly in print, and not online contexts ${ }^{2,3}$.

In an attempt to prove apparent banner blindness, Dreze and Hussherr ${ }^{1}$ claimed that online viewers may be aware of banners on a webpage since they shared "bandwidth with other elements of the page in which they are being displayed" (p. 10). As such, viewers may not look at the banners. Also, the viewers may have expected certain locations (e.g., horizontally across the top of the screen) to contain a banner. Their focus is therefore moved to content in other locations. Through eye-tracking, Lee and $\mathrm{Ahn}^{4}$ confirmed that banners with animation attracted less attention than static designs. However, the animation still unconsciously affects visitors' attitudes.

Empirical research has confirmed the banner blindness phenomena and indicated that online visitors do not pay equal attention to all the content on a screen ${ }^{5}$. In other words, viewers allocate their attention to online content in one location of the display more than in other locations. As a result, to draw the attention of the Y generation (age 18-31) viewers, website advertisers must include a sufficiently large image, especially of celebrities, and a search feature, while minimizing the amount of text on the screen ${ }^{5}$. Using an eye-tracking approach, viewers tended to scan a webpage in a top-down manner to locate cues for the brand they were looking for ${ }^{6}$. Similar patterns of webpage viewing were also found by Resnick and Albert ${ }^{7}$.

Despite the effects of banner blindness and the varying degree of attention online visitors pay to website content, there has been a recent practice by which ad banners appear at the bottom of a clip in a video-sharing website. For example, in Figure 1, the clip, as framed by a dotted line in Area B, is being watched while a banner, as framed by a grey line in Area C, appears at the bottom of the clip frame. Area A as framed by a thick dark line displays the other content, besides that in areas B and C. The clip in Figure 1 is a movie trailer for the film Die Hard 5 and the banner advertises a real-estate project in Bangkok, Thailand. Specifically, the banner is asking the viewer to take a certain action as indicated in the "register now" statement. The blurred region was intended to give contrast to Area 1 from the other areas and was only utilized for clarity.

The reasoning behind this placement strategy was that when the website visitors start watching the clip, they may fixate on the ad banner and eventually click on it to learn more. As a result, we considered it interesting to examine the correlations between fixation duration and fixation counts on the banner, or alternatively, fixation counts on the clip. To the author's knowledge, such empirical research has yet to be done. 


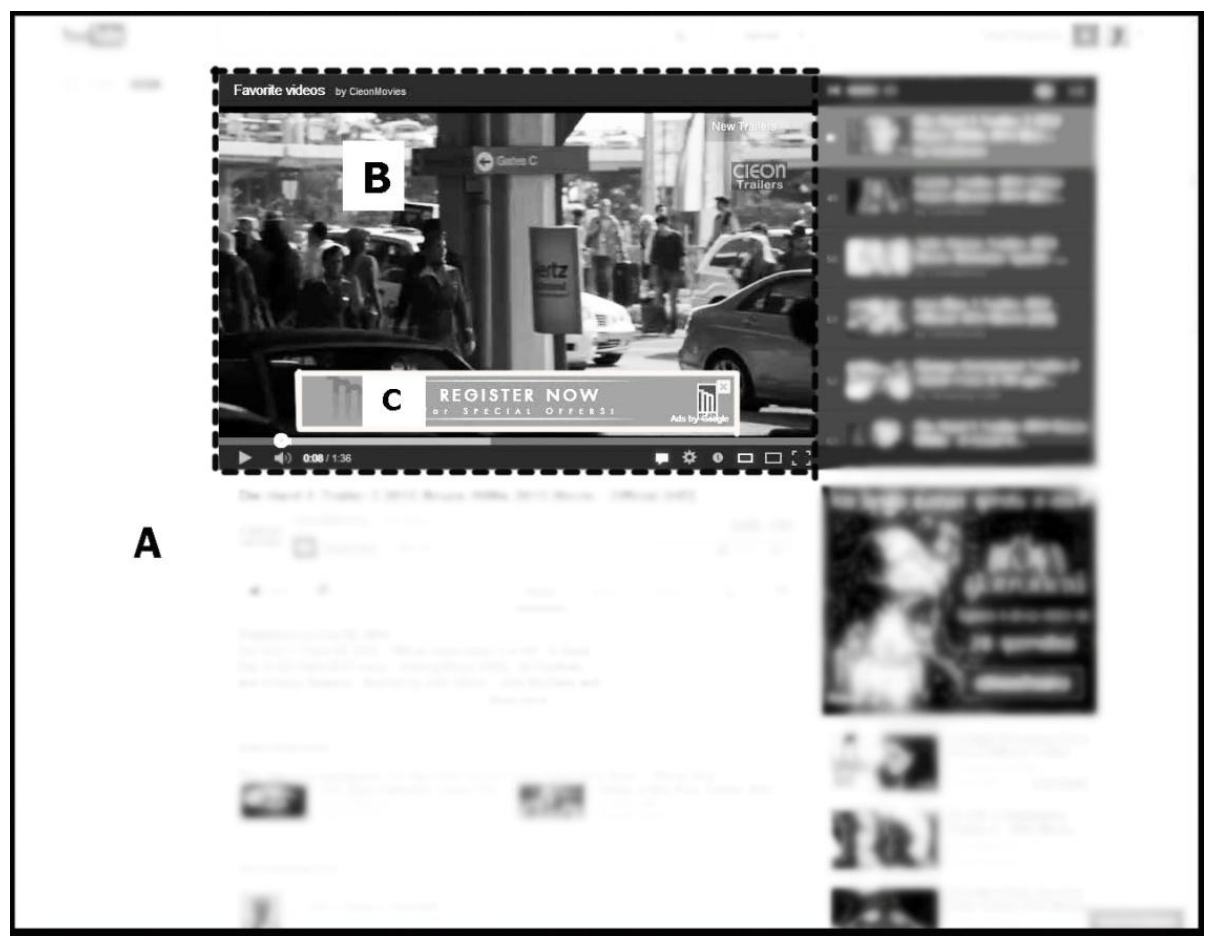

Figure 1. YouTube display of a trailer of the movie Die Hard 5 (Area B) featuring a banner (Area C) advertising a real estate project in Bangkok, Thailand

Among video-sharing websites, YouTube is listed in many sources as one of the most popular ${ }^{8,9,10}$. This remarkably high level of acceptance can be attributed to three components ${ }^{11}$. First, YouTube allows a display of videos with quality comparable to well-established video play technologies including Windows Media Player, RealPlayer or QuickTime. Users can upload videos of different formats which YouTube will subsequently convert to a uniform easily-playable format. Second, the actionable features of YouTube allow a straightforward sharing process. Furthermore, YouTube clips' HTML markup tags help users to easily embed them in other websites. Finally, YouTube assigns to a video clip a series of meta-data. The data includes: (1) typical identifiers such as a distinct 11-digit identification number, the date when the clip was added, or the uploader's identification; or (2) a list of related videos. Unlike videos distributed by peer-to-peer services, YouTube videos contain "relationship" attributes that provide a link to other videos with similar descriptions chosen by uploaders. According to Cheng et al. ${ }^{11,12}$, these three features account for the significant level of acceptance YouTube has earned. For these reasons, YouTube has been chosen as the context of the current study. 
Among indicators of advertising effectiveness, recall is of principal interest in this study. Typically, online advertisers expect that viewers should be able to recall what they have seen on a computer screen in an online campaign. The greater the recall, the more effective the campaign is expected to be. A few empirical studies have confirmed that there is a small amount of recall of banner promotions amongst viewers ${ }^{3,4,13}$. However, virtually none of these studies has examined recall in the context of video clips placed on YouTube or in conjunction with viewers' fixation behaviors.

As a result, the four objectives of the present study are: (1) to examine whether viewers of YouTube clips have fixated on ad banners appearing at the bottom of clips, (2) to determine what correlation exists between fixation duration on banners and fixation counts, in addition to (3) the correlation between fixation duration on banners and fixation counts on clips, and finally, (4) the extent to which visitors recalled content in the banners, and details of the clips.

The remaining sections are outlined as follows: the literature review addresses findings of previous research on eye-tracking and electronic commerce and will include a discussion of the rationale behind the current study; the third section explains the research methodology followed by a report of the results; the final section provides conclusions, including the contribution of the findings to the field and study limitations.

\section{LITERATURE REVIEW}

\subsection{Eye-Tracking Method}

Tracking what a person visually fixates on is challenging. Subjects' responses to a questionnaire about content viewed points to issues of validity and reliability of eye-tracking in research. These issues include: inaccuracy in recall of what subjects claim to see and secondly, lack of recognition of what was seen. However, advances in eye-tracking technology are able to provide some valid insights. The moment at which people see something may coincide with a cognitive gap for which they require further information to cope. Basically, an eye-tracking device records a subject's eye movement as being exposed to visual stimuli and aggregates the data in both qualitative (e.g., footage of what is looked at) and quantitative ways (e.g., coordinates of areas of fixation). The process of tracking how a person watches items on a computer screen involves two issues: saccades and fixation. The saccade is the simultaneous movement of the eyes in the same direction as it jumps from one fixation point to another. Although simultaneous, the eyes must pause on a specific area of the screen and this is known as the fixation. In order to piece together a complete 
image at which one is looking, an individual will fixate on one spot and then move the gaze. The image of the fixation takes place in one's fovea. It provides a highly detailed, clear image of what one is focusing on. Given the tiny area of fixation, one must shift one's gaze in saccades around the area of interest in order to fully form an image.

As a result, an eye-tracker will record the location on which a person fixates and track the movement of his or her eyes. Figure 2 shows the outcome from an eye-tracker of a subject's eye movements during a visit to a Wikipedia webpage. This viewer's first fixation was on the navigation section labeled Number 1 . The next fixation point, together with the saccadic path, were also recorded. Researchers often pay attention to the cluster of recorded fixations in a particular region since they provide valid evidence of a viewer's visual attention ${ }^{14}$.

While Figure 2 provides a qualitative summary of a visual representation of how an eye-tracker records fixating behavior, it is not the exact data actually recorded primarily by an eye-tracker. In fact, the tracker adopts a screen mapping coordination approach, that is, what a person fixates on a screen is observed and recorded as an $(\mathrm{x}, \mathrm{y})$ coordinate. The Miramatrix eye-tracker, for example, considers a computer screen as a map of $(\mathrm{x}, \mathrm{y})$ coordinates where the screen's upper left corner is deemed the $(0,0)$ coordinate, and the $\mathrm{X}$ axis represents the screen's vertical edge while the $\mathrm{Y}$ axis represents its horizontal edge. This is a unique definition that users of the Miramatrix eye-tracker must be aware of since it is in the opposite of the typical definition in which $\mathrm{X}$ and $\mathrm{Y}$ denote vertical and horizontal axes. A visual representation of this mapping is given in Figure 3.

With the coordinate mapping technique, researchers are allowed to define an area of interest (AOI). The AOI is a certain area on a computer screen which researchers define by shape in an attempt to explore whether a fixation occurs in the area ${ }^{14}$. To declare the AOI, researchers adopt the coordinate format. For example, one could define an AOI in Figure 4 surrounded by the four coordinates as $(140,870),(1000,870),(1000,990)$ and $(140,990)$. In a usability assessment, it is important to detect if viewers have fixated on a given AOI. Olmsted-Hawala \& Bergstrom ${ }^{15}$ were able to adjust the display of fields on an online form based on the samples' fixations on particular fields that were defined as AOIs in their study.

In the present study, we identified two AOIs on which to analyze fixations. The first AOI is the banner that appears at the bottom of a video clip and the second is the exclusive display of the clip. Note that the former embeds in the latter. When we refer to the clip, it thus means the display area of the clip with the banner removed. Referring to Figure 1, our first 
AOI is Area C and the second is Area B with Area C removed. Irwin ${ }^{16}$ recommended two variables associated with an AOI: fixation duration and fixation counts. The two constructs are valid indices of cognitive processing 16,28

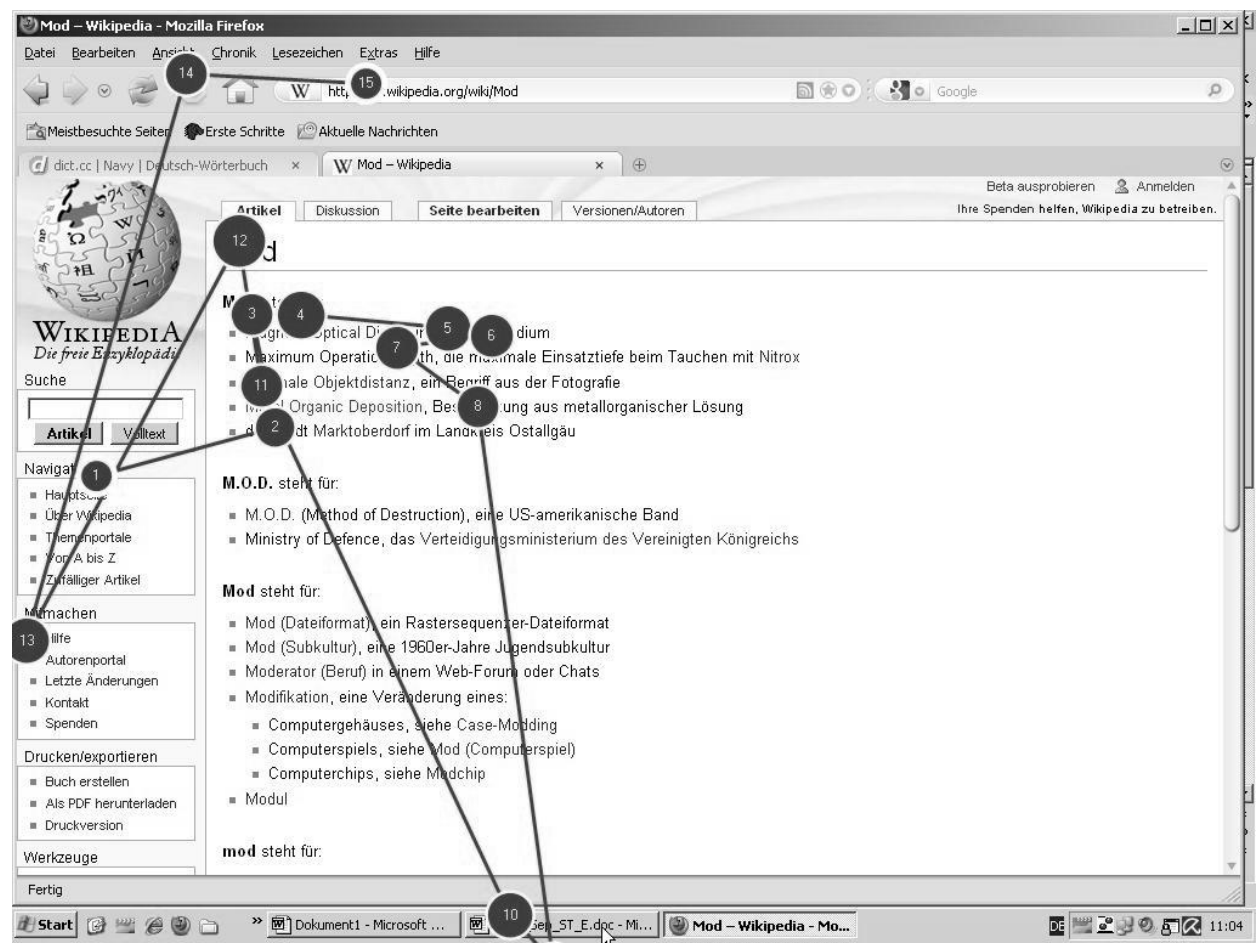

Figure 2. An example of fixation points on Wikipedia (http://ilcea.revues.org/docannexe/image/1060/img-3.jpg, accessed on September 24, 2014)

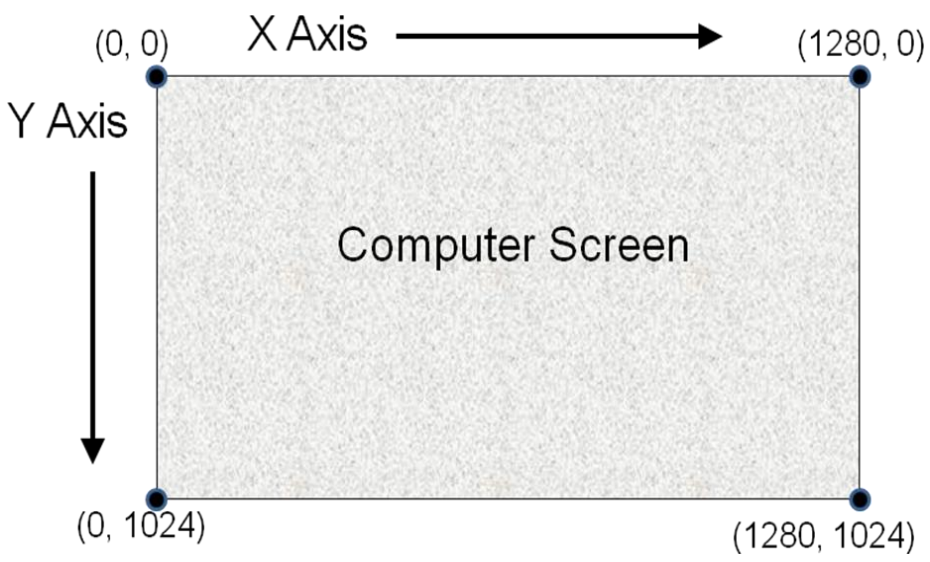

Figure 3. Mapping coordinates on a screen display 
One fixation refers to one pause recorded by an eye-tracker to confirm that a viewer has fixated once on an area of interest (AOI). Given a coordinate of a fixation, researchers are able to determine whether the fixation is in the AOI. For example, if the eye-tracker records the two coordinates as a subject is visiting the webpage in Figure 4 as $(132,750)$ and $(215,930)$, we could have a program script to detect that the former is not in the specified AOI, but the latter is. Bergstorm and Schall ${ }^{14}$ contend that a fixation is the eyes' brief rest on a certain area during saccadic movement. It is possible that in a video viewing session, one may fixate on and off the video clip. Consequently, fixation counts refer to the total number of recorded fixations on a given AOI in one session and the fixation duration refers to the total amount of time (in milliseconds) during which an eye-tracker records the viewer's fixation on a specific AOI.

In addition to studies of advertising on a computer screen, eye-tracking can be applied in many contexts including landscape architecture and printing technology. Prior to the data collection section, a researcher typically needs to calibrate the device, after which the actual tracking process can begin. The process is non-intrusive and can be applied to any person including those wearing glasses or contact lenses. However, on occasion, the subject may wear rather thick lenses or may have oddly-shaped eye balls making calibration of the tracking device impossible. Such subjects were therefore excluded from the current study.

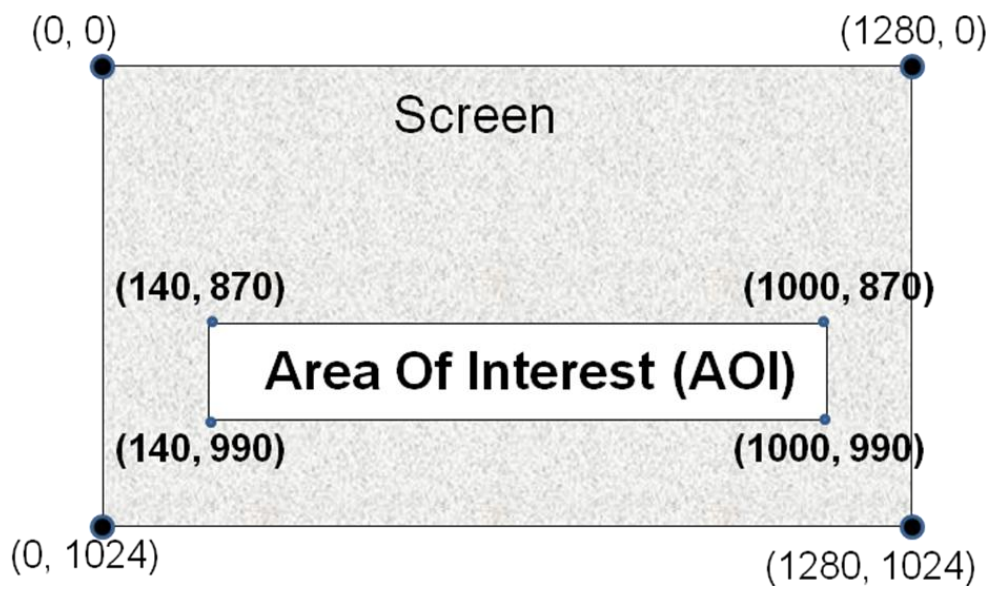

Figure 4. Mapping coordinates on a screen display with one AOI identified

\subsection{Banner Blindness}

A large volume of findings in previous research has verified the phenomenon of banner blindness $1,18,19,20$. Banner blindness is commonly 
defined as the absence of fixation on banners ${ }^{3}$. Yet, Hervet et al. ${ }^{3}$ used an eye-tracking device to prove that $82 \%$ of subjects fixated on banners at least once. They claim that viewers were not blind to banners, but instead they sometimes deliberately avoided fixating on them. Resnick and Albert ${ }^{7}$ confirmed based on their eye-tracking experiment that viewers who had specific goals when visiting websites were less likely to fixate on ad banners than those who browsed web pages for non-specific purposes. In other words, banner blindness is less evident in a browsing visit than with a webpage viewer pursuing a specific task.

Ad banners often compete for viewers' attention with other content on the same webpage. A number of studies have therefore examined banner design features that could lead to increased attention or secure viewers' fixation $^{2,21}$. Lee ${ }^{22}$ contended that website visitors became blind to banners due to information overload. According to this idea, in response to continuous information flow, online viewers simply ignore much of the information they encounter. They pay attention only to what they consider "highly relevant" to themselves. As such, what is obvious to some may be invisible to others. In addition, banners located at the top of a computer screen were relatively more noticeable than those at the right of the screen ${ }^{5}$. Combining the habituation-tedium theory and ergonomics domains, Portnoy and Marchionini ${ }^{23}$ claim that banner blindness is not solely the result of viewers' intention to ignore the banner, but also depends on the frequency with which viewers have previously encountered banners. During search sessions, viewers are not equally aware of their online surroundings. Consequently, they could exhibit inattention to banners because (1) the stimuli did not draw their attention or (2) they intended to ignore the stimuli $^{23,24}$. Cho and Cheon ${ }^{19}$ examined why people avoided advertising on the Internet and found that perceived goal impediment is a significant antecedent of advertisement avoidance.

\subsection{Examination of Fixation and Recall}

Online advertising campaigns often rely on visual stimuli. Consequently, advertisement designers assume that stimuli will be able to initiate viewers' fixation, and retain their attention. In a print ad medium, researchers ${ }^{25,26}$ used eye-tracking to confirm that a greater fixation rate of magazine readers correlated with better brand recall. Seo et al. ${ }^{27}$ noted the significant effects of brand image on visual attention in a Korean electronic commerce website.

Use of an eye-tracking device allows researchers to accurately calculate the number of fixations in an AOI. A single count is determined when a visitor starts gazing on a banner (Area $C$ in Figure 1) until their 
fixation moves out of the banner area. As such, if a viewer fixates on and off the banner on a screen, this could increase the number of fixation counts in one session. Researchers may count fixations on an ad banner to quantify the extent to which a web viewer has seen it. Through eye-tracking, Hervet et al. $^{3}$ verified that about 9 in 10 subjects had fixated on an ad banner at least once. Based on these studies, it appears the actual occurrence of banner blindness is low. The location in which a banner appears on a screen and the degree of animation in the banner content has an interactive effect on the fixation count on banners ${ }^{28}$.

Previous research has attempted to examine factors that contribute to the amount of attention spent in fixation and high fixation counts. Through eye-tracking, it has been shown that facial images attract higher fixation counts than corporate $\operatorname{logos}$ in a financial service website ${ }^{29}$. Seo et al. ${ }^{27}$ discovered that famous personalities (what they call "human brand image") in an endorsement on an electronic commerce website were able to attract longer durations of fixation than typical presenters. An eye-tracking analysis of a recruitment website revealed that Internet-based job seekers had a higher number of fixations on text than on graphic images ${ }^{30}$. These findings imply that online viewers fixate on particular digital details more than others. Hamborg et al. ${ }^{13}$ confirm that animated banners were able to trigger viewers' fixation better, thus leading to better recall of advertised products and their details than static ones. Fifty-five percent of viewers fixated at least once on static banners, while 94\% fixated on animated banners. However, $62 \%$ of viewers who responded to survey questionnaires falsely responded that they had not noticed any banner on the experimental website. Using an eye-tracking technique, Simola et al. ${ }^{31}$ verified that animation of ad content and abruptly appearing ads could draw viewers' attention. This distraction was evident in both browsing and reading tasks. According to the review by Higgins et al. ${ }^{32}$, the distraction may consequently draw viewers' attention, yet no empirical evidence confirms this statement.

In the context of search engines, the location for display of content is critical (i.e., whether it is on the top, right or at the bottom of the screen), given the large number of stakeholders on a webpage displaying search results. The fixation counts on content at the bottom of a page should be fewer than those at the top of the page. This speculation was empirically confirmed by Guan and Cutrell ${ }^{33}$. In fact, Goldberg et al. ${ }^{34}$ verified through eye-tracking that users of search engines process information on the results page from left to right in the same column. Guan and Cutrell ${ }^{33}$ further remark that the results of search engine optimization and those of sponsored items may receive different fixation counts. The conclusion to draw from these studies is that a search engine web designer must be very careful in 
displaying various types of content on a search result page since viewers fixate only on some items and may miss others.

In addition to fixation counts, fixation duration is another interesting research construct. It is defined as the amount of time a viewer spends fixating on a banner. Few studies have addressed fixation on ad banners ${ }^{18,22,}$ 27. Among the few who have is $\mathrm{Lapa}^{21}$ who found that fixation duration decreased when the format of successive web pages was the same. Given identical layout, Internet users may learn quickly to refrain from looking at the same screen area as they are aware of what to expect from previously viewed screens.

As seen in Figure 1, content on the screen can be classified into three types based on the three major locations of display: the ad banner (Area $\mathrm{C}$ ), the video clip (Area B excluding the ad banner) and the rest of the screen (Area A). Presenting an ad banner on a clip is a common service on a video-sharing website. While viewers enjoy the clip, they are assumed to have seen the banner. We believe that the fixation duration on the banner should be long enough to attain high fixation counts if an ad banner is to be effective in retaining the attention of viewers. Consequently, Hypothesis one is that the relationship between fixation duration and the fixation count is statistically significant. Although such a relationship may be anticipated, there is, as yet, no empirical evidence to validate it.

In general, web-based advertising and ad banners compete with other content on the same page for viewers' attention. On video-sharing websites, the ad banner is perceived as complementing and not competing for viewers' attention with the clip since the placement of the former is embedded in the latter. Hypothesis two is therefore the correlation between fixation duration on the ad banner and the fixation counts on the YouTube clip itself is statistically significant. Besides the clip and the ad banner, other content on a YouTube screen may also play a role in online marketing strategy. Intuitively, it is understood that other content should be plain so as not to draw viewers' attention which should focus mainly on the ad banner or the clip.

The rate of fixation on a YouTube ad banner is not the only indicator of online advertising success. Online marketing practitioners are often interested in examining if visitors are able to recall the content of banners. As such, for purposes of this study, ad content recall is considered to be the extent to which a visitor can remember the details of a banner and recall them without aids or prompts. Given that the banner is supposed to compliment the clip, we wanted to see to what extent viewers can also recall details associated with the clip. Examining whether viewers can recall clip 
and banner content is consistent with Till and Baack's method ${ }^{35}$ used to investigate the effects of creative television commercials. According to Hamborg et al. ${ }^{13}$, about $11 \%$ of site visitors were able to correctly recall products on banners but none were able to recall written details. Similarly, only $8 \%$ of viewers correctly identified whether an ad banner had been present $^{30}$. Moreover, Bayles and Chaparro ${ }^{7}$ discovered that less than $50 \%$ of viewers could recall the presence of ad banners. Based on an eye-tracking examination, Kuisma et al. ${ }^{28}$ confirmed the effect of interaction between animation of ad content and ad format on viewers' attention measured by fixation counts. Animated ads displayed vertically garnered the most attention. Furthermore, animation was found to significantly improve recall of content. Kuisma et al. ${ }^{28}$ discovered one unexpected finding. Their subjects were able to recognize ad content without actually having to look at the ad (i.e., there was no fixation on the ad). They further discuss ways in which online consumers are able to allocate their focal attention to irrelevant ads when they are involved in multiple tasks on a screen.

Recall can be measured in two ways: unaided and aided. While the former is to test if a person can properly describe details of a stimulus, the latter is to assess if the person can confirm the stimulus once it is presented. Aided recall is thus similar to recognition ${ }^{36,37}$. Consequently, the present study focuses on unaided recall. Moreover, an effective advertising project should attract viewers' attention and reinforce the ability to recall details of advertisements.

\section{METHODOLOGY}

\subsection{Population and Participants}

The target population of this study were people who have watched a clip on YouTube at least once. Since Chulalongkorn Business School in Chulalongkorn University allowed the authors to use an eye-tracking device at a university lab, the subjects chosen for the research were undergraduates from the business school. Thus, no claim is made that the college students chosen as subjects in the study represent the target population. However, a large proportion of those who are active users of video-sharing websites are in the same age range of the chosen participants in the current study. According to a 2010 survey by comScore, people between 15-24 years old were the largest group of viewers (as compared to other age groups) and spent an average of 42.1 hours per month watching online videos ${ }^{38}$. Two years later, the comScore survey showed that $90 \%$ of Internet users were young people in this age group each of whom reported to watch an average of 202.5 videos on the Internet ${ }^{39,40}$. General Internet user age profiles 
provide support that users in the 15-24 year age range represent a significant portion of current Internet users. For example, the largest group (20\%) of Internet users in Thailand are 15-24 years of age ${ }^{41}$. A 2013 survey by Pew Research shows that the largest (30\%) group of Internet users worldwide is between 13-25 years of age ${ }^{42}$. We therefore believe that those selected to participate in the current study represent the largest group of Internet users including those who enjoy watching videos online.

We announced a call for research participants in two undergraduate and two graduate classes at Chulalongkorn University. A total of 107 students participated voluntarily in the study. Some of the subjects were rewarded extra points for class, but they learned of this only after completing the participation. As such, their participation was voluntary. Table 1 presents a profile of the participants. Just under $30 \%$ of subjects were men, $42 \%$ were $18-19$ years old while $68 \%$ were undergraduates. Only $37 \%$ of the participants had normal vision.

Table 1. Participant profiles $(\mathrm{n}=103)$

\begin{tabular}{lll}
\hline Profile & Category & $\mathrm{N}(\%)$ \\
\hline \multirow{2}{*}{ Gender } & Male & $28(27)$ \\
& Female & $72(73)$ \\
\hline \multirow{2}{*}{ Age } & $18-19$ & $43(42)$ \\
& $20-25$ & $48(47)$ \\
\multirow{2}{*}{ Education } & $>25$ & $12(11)$ \\
\hline \multirow{2}{*}{ Subject vision } & Undergraduate & $70(68)$ \\
& Graduate & $33(32)$ \\
\hline
\end{tabular}

\subsection{Variable Operationalization}

The six major variables in this study were: (1) whether YouTube viewers fixate on an ad banner placed at the bottom of a YouTube clip, (2) the duration of fixation on the ad banner, (3) fixation counts on the ad banner, (4) fixation counts on the YouTube clip and (5) recall of what was advertised on the banner (banner recall) and (6) recall of content featured on the clip (clip recall).

A Miramatrix eye-tracking device was used to verify whether visitors gazed on an ad banner and measured the duration (in milliseconds) that the viewer fixated on the banner. In addition, eye-tracking was used to count the actual number of viewer fixations on a banner and clip. The Miramatrix s2 system was connected to a desktop computer with a resolution of $1024 \mathrm{x}$ 768 pixels. The eye-tracker's resolution and sampling rate were at most $1^{\circ}$ 
and $60 \mathrm{~Hz}$, respectively. Eye movements were captured by a camera placed beneath a $17 "$ computer screen located about $50 \mathrm{~cm}$ from the participant.

The use of the eye-tracker helps us to reliably and validly detect a viewer's fixation on a screen. Had the viewer been asked using a questionnaire, their response may have been subjective. It is uncommon that a person who has enjoyed a YouTube clip would be able to reliably recall whether they had looked at a specific banner ${ }^{14}$.

Given that our participants were college students, we strove to select movie trailers that would be of interest to that segment of viewers. The visual stimuli selected were thus three clips on YouTube viz. movie trailers of Die Hard 5, The Smurfs 2 and one supplied from the channel Cieon, a YouTube clip supplier. Die Hard 5 and The Smurfs 2 were selected to represent the two genres of action and animation movies, respectively. The third clip was selected from the Cieon collection. We selected the clip from Cieon to ensure a variety of movies for the rest of the participants. There are three reasons why we selected these clips. First, we want our findings to be generalizable to the population of users in this age group. This is why using only one clip was deemed inadequate. Second, we chose to use clips of comparable length on which one ad banner would appear. Each of the three clips lasted between 1.5 - 2 minutes, a sufficient period for participants to enjoy the content of the clip and to track their gaze. In other words, this length of time was considered sufficient to observe whether subjects fixated on the clip or on the ad banner, but was not too long for research purposes. Third, all clips selected had banners of comparable size located in the same location. Figure 5 shows two sets of four coordinates that define two AOIs in the current study. The first set $\left(\mathrm{cx}_{\mathrm{i}}, \mathrm{cy}_{\mathrm{i}}\right)$ where $\mathrm{i}=1,2,3,4$ is for the location of the clip and the second $\left(b_{i}, b_{i}\right)$ where $i=1,2,3,4$ is for the ad banner. For instance, the Die Hard 5 clip fits within coordinates $(225,136)$, $(225,560),(866,136)$, and $(866,560)$. Although the other clips chosen may not have exactly the same coordinates as those in Die Hard 5, they were of comparable size and location.

Though all clips were in English, we do not believe this posed a problem for the subjects (Thais) to follow them since they were brief. During the three-day observation before the actual data collection, all three selected clips met our requirement criteria; all three were therefore kept for use in the current study.

The decisions regarding the movie selections were mainly to enhance the finding's reliability. For instance, the selected movie clips must be of varying interest to the sample. Consequently, we opted for clips available through the Cieon collection, in addition to the action (i.e., Die Hard 5) and 
animation (The Smurfs 2) movie clips. Also, our selection of clips of comparable length (i.e., 1.5 - 2.0 minutes) had implications on the study reliability. Had we selected clips of far different lengths, the audience may not have recalled viewing the banners and the movie clips.

Banner and clip recall measurements were unaided. After viewing the YouTube clip, subjects were asked if they remembered which product was in the banner. In fact, they were asked to write down the advertised product name. Unaided recall was used because we wanted to test viewers' memory of banner content which was measured as banner recall. This was the first step before testing for other aspects of memory ${ }^{35}$.

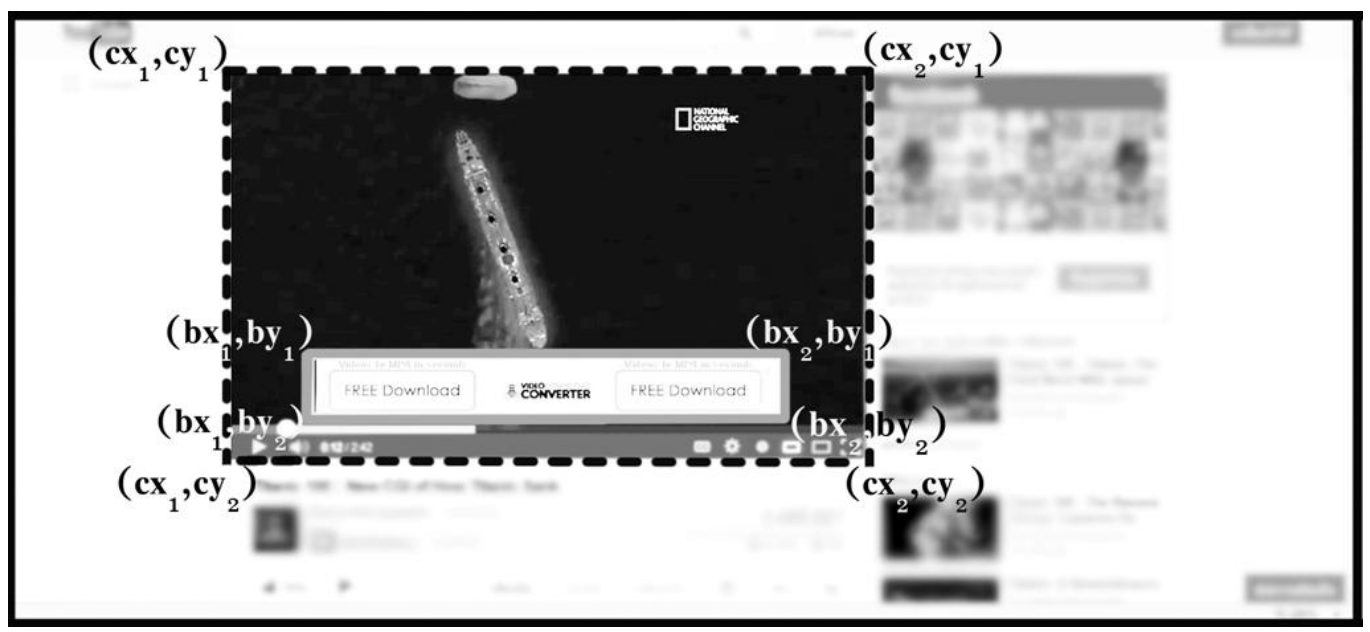

Figure 5. Coordinate references on the current study's clip (cxi, cyi), where $\mathrm{i}=1,2,3,4$ and those of the ad banner (bxi, byi), where $\mathrm{i}=1,2,3,4$

Once subjects responded to the banner recall question, they were asked to answer two additional questions that measured recall of the clip. In the two questions, we asked the subjects to write (1) the title of the movie clip and (2) the day the movie was to open in theatres. The subjects earned 0 points if both answers were incorrect, 1 point if partially correct and 2 points if both were correct. The two questions were intended to measure accuracy of recall of the clips.

Note that the data in the current study were collected in a captive environment. We required that a subject watch the YouTube clip while eye-tracked, after which the subject responded to a few questions. A typical research design with a pretest or control group was not employed. However, researchers adopting an eye-tracking approach often refer to such data collection as an experiment ${ }^{14}$. 


\subsection{Procedures and Analysis}

Once participants arrived at the lab, they received a short note explaining the purpose of the research and importance of their participation. The participants were asked to take a look at a movie trailer on YouTube, after which they were asked to respond to a brief questionnaire. We did not mention about the ad banner. Although they were not directly asked for a consent, the participants learned from the note that they were free at any time to leave the lab, if uncomfortable with the circumstances.

When they were ready, the eye-tracking device was calibrated to each participant, which took about $1-1.5$ minutes after which the participant started viewing the YouTube clip. The aim behind the calibration for each participant was to ensure instrument reliability. Participants were randomly selected to watch one of the three clips. While viewing, the subject's eye movements were recorded for further analyses. After the clip ended, they were asked to answer the recall questions, and then filled in their demographic information and received a ballpoint pen for their contribution to the study. Of 107 students that signed up to participate in the study, four did not show up, leaving 103 usable records. We then processed the data in order to derive the values of the six major variables. A research assistant examined the data recorded in the eye-tracker in order to (1) check whether the participant fixated on a banner and (2) to count the number and measure the duration of fixations on the banner and count the number of fixations on the clip. The assistant subsequently combined the fixation data and the recall data to form the data set for further analysis and hypothesis testing.

Descriptive statistics of all variables were recorded. The analyses of fixation and recall were mainly done through percentages and cross tabulation. Moreover, the Pearson's r correlation was used to test the two hypotheses only if the fixation duration and fixation counts were normally distributed. If not, non-parametric correlation replaced the Pearson's r.

\section{RESULTS}

As seen in Table 2, 97\% of subjects fixated at least once on the YouTube ad banner. The analysis on the minors who had not fixated on the ad banner indicated that their focus was neither on the ad banner nor the clip but on the content in other areas. This further implies that in this study, there was no banner blindness ${ }^{3}$. The subsequent analyses are therefore based on the 100 samples who we found through eye-tracking to have gazed on the ad banner at least once. As seen in Table 3, among those who had fixated on the ad banner, only $8 \%$ were able to correctly recall what was advertised on 
the banner. However, when asked about the clip, 6 out of 10 could give the correct movie title and 5 in 10 were able to recall the correct air time. In short, about $50-60 \%$ of the subjects were able to correctly recall the clip details while $8 \%$ could remember the clip titles properly.

Table 2. Fixations on YouTube Ad Banner $(\mathrm{n}=103)$

\begin{tabular}{lc}
\hline Fixations & $\mathrm{N}(\%)$ \\
\hline No Fixation & $3(3)$ \\
At least one fixation & $100(97)$ \\
\hline
\end{tabular}

As shown in Table 4, the participants fixated on the ad banner for an average of 573.01 milliseconds. The average number of fixations on the ad banner and on the YouTube clip were 17.88, and 73.96, respectively. Based on skewness and kurtosis, we conclude that the variables were not normally distributed since their absolute values exceeded one ${ }^{43}$. As a result, the correlation analysis had to be non-parametric. We thus substituted Pearson's $r$ with Spearman's rank correlation.

Table 3. Content recall of clips and banners viewed $(n=100)$

\begin{tabular}{lc}
\hline Content & $\mathrm{N}(\%)$ \\
\hline Banner Ad & \\
Correct recall & $8(8)$ \\
Incorrect recall & $92(92)$ \\
\hline Movie clip titles & \\
Correct recall & $58(58)$ \\
Partially correct recall & $36(36)$ \\
Incorrect recall & $6(6)$ \\
\hline Movie clip details & \\
Correct recall & $43(43)$ \\
Partially correct recall & $10(10)$ \\
Incorrect recall & $47(47)$ \\
\hline
\end{tabular}

In the first hypothesis we attempted to test if the correlation between duration of fixation on an ad banner and fixation counts on the banner was significant. Yet, the correlation found $(-.100, p<.324)$, shown in Table 5, was insignificant. For the second hypothesis, the correlation between duration of fixation on the ad banner and fixation counts on the clip was negatively significant $(-.486, p<.000)$. The following section provides further discussion of the findings. 
Table 4. Descriptive statistics for variables $(n=100)$

\begin{tabular}{lcccc}
\hline Variable & Mean & Standard deviation & Skewness & Kurtosis \\
\hline $\begin{array}{l}\text { Duration of fixation on ad banner } \\
\text { (milliseconds) }\end{array}$ & 573.01 & 0.22 & 1.02 & 1.26 \\
Fixations on ad banner (times) & 17.88 & 13.81 & 1.46 & 2.71 \\
Fixations on YouTube clip (times) & 73.96 & 33.60 & 0.75 & 0.10 \\
\hline
\end{tabular}

Table 5. Correlation matrix using Spearman's rank correlation analysis

\begin{tabular}{lc}
\hline & Duration of fixation on ad banner \\
\hline Fixations on the ad banner & -.100 \\
& $(.324)$ \\
Fixations on the YouTube clip & -.486 \\
& $(.000)$ \\
\hline
\end{tabular}

Note: *in parentheses are p-value above which are correlation coefficients.

\section{CONCLUSION AND DISCUSSION}

This research used an eye-tracking approach to explore YouTube viewers' fixation on and recall of an ad banner placed on YouTube movie clips. A total of 103 students from the Chulalongkorn Business School were selected randomly to view one of three movie clips. While viewing, their eye movements were tracked, recorded and converted into a count of fixations on ad banners and video clips. Despite the relatively small size of the sample, as college students are in the age range of a large portion of both Internet users and YouTube viewers ${ }^{32,42}$, findings based on this group of subjects are deemed to have validity.

Through eye-tracking, it was shown that $97 \%$ of the subjects fixated at least once on the ad banner placed at the bottom of YouTube clips. This is confirmation of the scant chance of banner blindness if that term means complete lack of fixation on a banner. Compared to the number of fixations on ad banners reported in previous research ${ }^{13}$, the rate in the present study (97\%) was found to be similar to the rate of fixations on animated banners (94\%), but considerably higher than that on static banners (55\%) reported in the same experiment. Such a high figure may suggest that placing an ad banner on a YouTube clip could be a better strategy than presenting it via other channels. In addition, a sizable number of studies have reported banner blindness under different conditions which included undertaking various tasks during a visit to a website, or alternatively, a number of banners appearing in different locations on the webpage in question ${ }^{35,44}$. 
Our attempt may be the first to examine banner placement on YouTube clips and we were able to show that nearly all viewers fixated at least once on the banner in this location. It is thus reasonable to claim that embedding a banner on a YouTube clip is a relatively feasible strategy for some types of advertising. The no-blindness incidence may be accounted for by the type of task undertaken during a visit to YouTube. Previous work has suggested that viewers whose purpose of a visit is to browse items online are more likely to notice and fixate on a banner in the website than those with another, more specific goal ${ }^{7,28}$. People presumably visit YouTube to relax and enjoy watching video or movie clips. YouTube viewers are therefore likely to notice a banner embedded in such a clip.

We report that our research subjects on average spent .573 seconds gazing on an ad banner on a YouTube clip. In other words, the viewer allocated about half a second to fixate on the banner. In terms of the number of fixations on the banner and on the YouTube clip, it was found that subjects fixated on average 17.88 and 73.96 times, respectively. Again, subjects in this study, at least, were not blind to the ad banners. Compared to the findings in previous projects ${ }^{22,} 45$, the number of fixations and the duration of fixation on banners in Koster et al. ${ }^{45}$ were 4.02 times and 0.851 seconds while those in $\mathrm{Lapa}^{21}$ were 3.01 times and 2.69 seconds. Our finding concerning the average fixation count (i.e., 17.88 times) was considerably higher, but that of the average fixation duration (i.e., 0.573 seconds) was considerably lower.

In Koster et al. ${ }^{45}$, personalized banners obtained more fixation counts than non-personalized ones. Similarly, in Lapa ${ }^{21}$, banners with content related and relevant to that on the webpage on which the banners were displayed received more fixation counts than those with unrelated irrelevant content. The more frequent fixations and the shorter length of each fixation in our study may indicate a poor match in content between the banner and the movie clip. As such, the viewers might not have wanted to spend time looking at them. Also, the number of fixations on the YouTube clips was roughly four times higher than on the ad banner. It is reasonable to assume in the current study that the clips were therefore considered more engaging to viewers than the ad banner. Had the banner been personalized or had content been relevant to the webpage matter ${ }^{21,45}$, fixation on the banner would have been more frequent or of a longer duration. The significant negative correlation between the number of fixations on the clip and the fixation duration on the ad banner also supports the assertion that the clips were of greater interest to subjects than the ad banner. Nonetheless, as we are aware of no previous empirical work investigating issues about fixation 
on YouTube clips, and as such, there is no benchmark against which to compare these figures. This suggests an area for future research.

Among those who fixated on the ad banner in this study, only $8 \%$ were able to recall what was presented on the banner. Although $8 \%$ is a relatively low percentage, it is consistent with results in previous studies ${ }^{13,46}$ in which about $8-11 \%$ of viewers were able to correctly recall the presence of banners or their content. According to Koster's work ${ }^{45}$, subjects were able to recall an average of $21.6 \%$ of all ad content. Such a high level of recall could have been due to their use of personalized banners. Once personalized, subjects could have recalled the ad better than in our study $(8 \%)$. In addition to banner recall, the subjects in the present study were able to recall details of the movie clip (e.g., title and air time) more accurately than banner content (see Table 3).

Given the study's exploratory nature, two possible explanations are posited for this difference in recall. First, most viewers may have a tendency to pay more attention to content in a relatively larger area of the screen than in a small area. Since the banner at the bottom of a clip is often much smaller than the clip, viewers may see and perceive the banner as an annoying peripheral object and this may hinder recall. A second explanation pertains to the matter of competing content, i.e., the ad message was recalled with less accuracy than the clip, perhaps because the clip content was much more engaging to watch. The second explanation is supported by the fact that the number of fixations on the clip was four times higher than on the ad banner and the significant negative correlation between fixation duration on an ad banner and number of fixations on the YouTube clip discovered in the present study. The longer that YouTube viewers fixated on the banner, the fewer fixations on the clip. If the clip content draws much of the viewers' fixation, they will necessarily spend less time focusing on the banner. In other words, had a less interesting clip been selected, it is possible that fixations on the ad banner would have been greater or could have led to greater recall of banner content. If the clip content is too uninteresting, however, no one would want to watch it, leaving the embedded ad banner unviewed. Consequently, the selection of an appropriate video clip is a challenge for online advertising designers. As these explanations are speculative, they require further research to validate.

From an online advertising perspective, the trivial correlation between fixation duration and fixations on ad banners is a disappointing finding in this study. Designers of online advertising might have expected a more significant positive correlation, i.e. in a successful YouTube advertising campaign, the higher the number of fixations on an ad banner, the longer the duration of each fixation. This could be assumed to be an indication of 
viewers' greater interest in the message on the ad banner. Although insignificant, the slim negative correlation coefficient in the current study might be a result of the message on the ad banner competing with the clip content for viewers' attention. While viewers were watching the clip, they could encounter some less engaging scenes during which their peripheral vision would pick up the ad banner embedded at the bottom of the clip. This should then lead viewers to fixate on the banner. Once a more engaging scene is encountered, interest on the banner might be interrupted. This is thus a matter of competing content between the YouTube clip and the embedded ad banner. The slightly negative relationship in the current study could indicate that the clips chosen were much more interesting than the ad banner. Viewers may have had a relatively high number of fixations (17.88) on the banner, but did not fixate on it long. In the review of the literature only the work of Koster et al. ${ }^{45}$ examined similar issues. The trivial correlation between the number of fixations on ad banners and the fixation duration found in the present study is consistent with what was found in Koster et al. ${ }^{45}$ However, as only two empirical research investigations came to this conclusion suggests further research is warranted.

The findings of this study have both theoretical and practical implications. Theoretically, the results have shed new light on the use of eye-tracking techniques for analysis of the effects on viewers of online advertising in the context of YouTube. Two major points of conceptual import stem from our findings. First, YouTube viewers are not blind to ad banners placed at the bottom of a movie clip, which is contrary to previous reports. However, they may not correctly recall banner content. Viewers nonetheless retain significant recall of movie content on YouTube clips. The second theoretical contribution is the finding of a significantly negative correlation between fixation duration on the ad banner and fixation counts on the YouTube clip. This confirms the competition for viewers' attention between the YouTube clip and the ad banner. The idea that YouTube viewers may enjoy a clip, notice the ad banner embedded at the bottom of the clip and ultimately click on the banner to further learn about the ad is not supported in the context of the present study. Such finding should prompt attention from other researchers and spur further studies to shed light on the topic.

These findings offer a practical contribution to two groups of stakeholders: online advertisement designers and clip-sharing website managers. As the people in charge of placing ad banners on a video clip, online ad designers have to conceive of a design that can secure the clip viewers' attention as well as accurate recall of the ad message. The present study's findings confirm the low probability of banner blindness if the ad 
banner is embedded at the bottom of a YouTube clip. Nearly all viewers in the study noticed the ad banner as demonstrated by having one or more fixations on it. Noticing the banner may have been a result of what was referred to by Hsieh et al. ${ }^{47}$ as "dishabituation." Typically, a web site visitor may assume the location of a banner and successfully avoid looking at it. However, if the presence of the ad banner does not conform to the viewer's expectation, they will be more likely to notice the banner. ${ }^{47}$ In other words, the dishabituation of a banner location could enhance a viewer's attention to the ad banner. According to Hsieh et al. ${ }^{47}$, an image or animation may better trigger the dishabituation effect and consequently yield more attention from the viewer as compared to plain text content. An alternative way to dishabituate website visitors expectations is to delay the display of an ad banner $^{31}$. According to Resnick and Albert ${ }^{7}$ and Simola ${ }^{31}$, viewers expect that the display of a webpage's top section will be faster than that of the bottom. While waiting for a webpage to download to the screen for example, if all sections excluding the banner appear on the screen, a viewer is likely to notice the location where the banner is being downloaded and subsequently wait to see the content of the banner.

In addition to viewers' awareness of advertisement message, designers of online advertising will desire successful recall of the ad content. The present study verified that YouTube visitors could not correctly recall what was on the banner, but their recall of details from the video clip was fairly accurate. Thus, designers of online advertising must adjust their campaign strategy to enhance recall of ad content. Findings in previous studies offer suggestions in this regard. First, animation in a banner enhanced viewers' recall of an online advertising campaign ${ }^{13,28,31}$. It was found that completely animated banners drew more of viewers' attention and resulted in greater recall than static banners. ${ }^{13}$ Moreover, animated ad content received a high number of fixations and relatively long fixation duration ${ }^{31}$. Interestingly, the number of clicks on animated banners did not differ from that on banners with a static design. As a result, designers of online advertising would be well advised to adopt animation in designs of ad banners since it could trigger recall, although it may not lead to a significant number of clicks. The subsequent challenge is to tailor online advertising by using the right amount of animation so as to achieve expected outcomes.

The other implication from the study is that ad personalization may trigger recall. According to Koster et al. ${ }^{45}$, personalized banners were able to trigger more recall of banner content than non-personalized ones. The banners in their study were embedded in a news website and personalized according to viewers' demographic characteristics including age, gender, interests, profession, and location ${ }^{45}$. Based on findings in Koster et al. ${ }^{45}$, 
online advertisement design could benefit by the inclusion of personalized ad banners making use of YouTube viewers' personal backgrounds prior to embedding in a video clip. Given competition for viewers' attention from content between the ad banner and the clip found in the current study, personalization of banner content may improve viewer attention on the banner over that reported in Koster et, al. ${ }^{45}$ As a result, designers of online advertising will have to balance personalization of banner design with the selected clip on which the banner will appear.

Website managers of clip-sharing websites such as YouTube are the second group of stakeholders who could benefit from the findings in the present study ${ }^{48}$. The aspect of key importance for managers is the selection of a clip that can successfully balance viewers' attention between the ad banner and clip content. There are two possible practical suggestions to achieve this. First, managers should be very well aware of the presentation of clip content to be able to pinpoint precisely the moment in a clip during which an ad banner should appear in order to balance competing content between ad and clip. For example, during a typical movie clip, there may be relatively less engaging moments during which the ad message could pop up to draw viewers' attention and retained until the scene draws the viewer's interest again. For example, in the 1:36-minute clip Frozen on YouTube (accessed on November 26,2014) the segment from 1:20 to 1:33 may be an opportune place for an ad banner since it is right after the climax of the clip, but still at a point where viewers are still watching to see the end of the clip.

Second, clip-sharing managers may work beneficially with online advertising planners on clip selection. Our findings confirm that a movie clip may engage viewers to the extent that they were unable to recall the content of the ad banner embedded on the clip, but still were able to recall clip details correctly despite most viewers fixating on the banner. According to findings from previous work, the point when an online viewer is most likely to notice an ad banner is while still browsing, and not while engaged in a particular site ${ }^{7,28}$. Should the chosen clip be a movie, it should have a moment where viewers could allocate attention to absorb an ad banner or the clip should be something other than a movie that will permit the viewer to notice the ad. For instance, the clip could be a documentary or routine presentation featuring a community leader attending a local event. Viewers of such routine types of content may focus on the presentation only moderately and still be able to allocate attention to absorb content from an ad banner embedded at the bottom of the display. Nonetheless, this is just a suggestion for which more empirical research is needed to confirm effectiveness. 
The study had three limitations. First, the context of Internet use is constantly changing. What can be found on YouTube today may not be valid on a future day. This imposes a limitation on the generalizability of the findings. More frequent research to keep up with changes is one remedy. Second, the data was collected in a captive environment. The subjects selected were those with access to a lab, and in the present case, college students. It is suggested that the use of eye-tracking methods in further advertising research extend to cover other types of online visitors or other websites. Given the exploratory nature of the current study, we excluded certain variables that may have confounded the recall of a banner on a video-sharing website. This could be another limitation of the current study findings. As such, future research could examine possible confounding effects such as a subject's disposition to a movie clip. The outcome of such research would further build on knowledge about online advertising on a website.

\section{ACKNOWLEDGEMENTS}

We are thankful for partial financial support from the "Chulalongkorn Academic Advancement into its Second Century" project. We also want to express appreciation for constructive comments from IJECS reviewers.

\section{REFERENCES}

[1] X. Dreze, and F. Hussherr, Internet advertising: Is anybody watching? Journal of Interactive Marketing, 17(4), p8-23, 2003.

[2] R.S. Moore, A. Stammerjohan, and R.A. Coulter, Banner advertiser-web site context congruity and color effects on attention and attitudes. Journal of Advertising, 34(2), p71-84, 2005. http://dx.doi.org/10.1080/00913367.2005.10639189.

[3] G. Hervet, K. Guerard, S. Tremblay, and S. Chatourou, Is banner blindness genuine? Eye-tracking internet text advertising. Applied Cognitive Psychology, 25(5), p708-716, 2011. http://dx.doi.org/10.1002/acp.1742.

[4] J. Lee, and J.-H. Ahn, Attention to banner ads and their effectiveness: An eye-tracking approach. International Journal of Electronic Commerce, 17(1), p119-137, 2012. http://dx.doi.org/10.2753/JEC1086-4415170105.

[5] S. Djamasbi, M. Siegel, and T. Tullis, Generation Y, web design, and eye-tracking. International Journal of Human-Computer Studies, 68(5), p307-323. 2010. http://dx.doi.org/10.1016/j.ijhcs.2009.12.006.

[6] E. Tzanidou, S. Minocha, and M. Petre, Applying eye tracking for 
usability evaluations of e-commerce sites. Paper presented at the workshop on 'Commercial Uses of Eye tracking' held at the 19th British HCI Group Annual Conference, Edingburgh, UK, September 5-9, 2005.

[7] M. Resnick, and W. Albert, The impact of advertising location and user task on the emergence of banner ad blindness: An eye-tracking study. International Journal of Human-Computer Interaction, 30(3), p206-218, 2014. http://dx.doi.org/10.1080/10447318.2013.847762.

[8] vidstatsx.com, YouTube Stats (Subscriber, Ranking, \& Video Statistics). Retrieved on October 25, 2014, from http://vidstatsx.com/youtube-top-100-most-subscribed-channels.

[9] www.alexa.com, Top sites. Retrieved on October 25, 2014, from http://www.alexa.com/topsites/category/Computers/Internet/On_the_W eb/Web_Applications/Video_Sharing.

[10] C. Lorenzo-Romero, M. Alarcon-del-Amo, and E. Constantinides, Determinants of use of social media tools in retailing sector. Journal of Theoretical and Applied Electronic Commerce Research, 9(1), p44-55, 2014. http://dx.doi.org/10.4067/S0718-18762014000100005.

[11] X. Cheng, C. Dale, and J. Liu, Statistics and social network of YouTube videos. Paper presented at the 16th International Workshop on Quality of Service, Enschede, June 2-4, 2008. http://dx.doi.org/10.1109/IWQOS.2008.32.

[12] X. Cheng, C. Dale, and J. Liu, Characteristics and potentials of YouTube: A measurement study, in peer-to-peer video. In E.M. Noam and L.M. Pupillo (Eds.), The Economics, policy, and culture of today's new mass medium (p205-227). New York: Springer Science, 2008.

[13] K.-C. Hamborg, M. Bruns, F. Ollermann, and K. Kasper, The effect of banner animation on fixation behavior and recall performance in search tasks. Computers in Human Behavior, 28(2), p576-582, 2011. http://dx.doi.org/10.1016/j.chb.2011.11.003.

[14] J.R. Bergstrom, and A.J. Schall, Eye tracking in user experience design. Boston: Elsevier, 2014.

[15] E. Olmsted-Hawala, and J.R. Bergstrom, Think-aloud protocols: Does age make a difference? Paper presented at the SIGCHI Conference on Human Factors in Computing Systems, Atlanta, GA, USA, April 10-15, 2010. http://dx.doi.org/10.1145/1753326.1753685.

[16] D. Irwin, Fixation location and fixation duration as indices of cognitive processing. In J.M Henderson and John M. (Eds.), The interface of language, vision, and action: Eye movement and visual world (p105-133). New York: Psychology press, 2004.

[17] M. Bayles, and B. Chaparro, Recall and recognition of statistic vs. animated banner advertisements. Paper presented at the Human Factors and Ergonomics Society Annual Meeting, Minneapolis, 
Minnesota, USA, October 8-12, 2001. http://dx.doi.org/10.1177/154193120104501510.

[18] M. Burke, A. Hornof, E. Nilsen, and N. Gorman, High-cost banner blindness: Ads increase perceived workload, hinder visual search and are forgotten. ACM Transactions on Computer-Human Interaction, 12(4), p423-445. 2005. http://dx.doi.org/10.1145/1121112.1121116.

[19] C.-H. Cho, and H.J. Cheon, Why do people avoid advertising on the Internet? Journal of Advertising, 33(4), p89-97, 2004. http://dx.doi.org/10.1080/00913367.2004.10639175.

[20] D.R. Fortin, and R.R. Dholakia, Interactivity and vividness effects on social presence and involvement with a web-based advertisement. Journal of Business Research, 58(3), p387-396, 2005. http://dx.doi.org/10.1016/S0148-2963(03)00106-1.

[21] C. Lapa, Using eye-tracking to understand banner blindness and improve website design. Unpublished master thesis in information technology. New York: Rochester Institute of Technology, 2007.

[22] C. Lee, Banner blindness: An effect of information overload on the World Wide Web. Retrieved on October 14, 2015 from http://www.carrielee.net.

[23] F. Portnoy, and G. Marchionini, Modeling the effect of habituation on banner blindness as a function of repetition and search type: Gap analysis for future work. Paper presented at the CHI'10 Extended Abstracts on Human Factors in Computing Systems, Atlanta, GA, USA, April 10-15. http://dx.doi.org/10.1145/1753846.1754142.

[24] C.Y. Yoo, K. Kim, and P.A. Stout, Assessing the effects of animation in online banner advertising: hierarchy of effects model. Journal of Interactive Marketing, 4(2), p49-60, 2004. http://dx.doi.org/10.1080/15252019.2004.10722087.

[25] W. Wedel, and R. Pieters, Eye fixation on advertisements and memory for brands: A model and findings. Marketing Science, 19(4), p297-312, 2000. http://dx.doi.org/10.1287/mksc.19.4.297.11794.

[26] R. Pieters, A review of eye-tracking research in marketing. In N.K. Malhotra (Ed.), Review of Marketing Research, Volume 4 (p123-147). United Kingdom: Emerald group publishing Limited, 2008. http://dx.doi.org/10.1108/S1548-6435(2008)0000004009.

[27] Y. W. Seo, S.W. Chae, and K.C. Lee, The impact of human brand image appeal on visual attention and purchase intentions at an e-commerce website. Intelligent Information and Database Systems, p1-9., 2012. http://dx.doi.org/10.1007/978-3-642-28493-9_1.

[28] J. Kuisma, J. Simola, L. Uusitalo, and A. Oorni, The effects of animation and format on the perception and memory of online advertising. Journal of Interactive Marketing, 24(4), p269-282, 2010. http://dx.doi.org/10.1016/j.intmar.2010.07.002. 
[29] S. Djamasbi, M. Siegel, T. Tullis, and R. Dai, Efficiency, trust, and visual appeal: Usability testing through eye-tracking. Paper presented at the 43rd Hawaii International Conference on System Science Proceedings, Honolulu, HI, January 5-8, 2010. http://dx.doi.org/10.1109/HICSS.2010.171.

[30] D. Allen, J.E. Biggane, M. Pitts, R. Otondo, and J.V. Scotter, Reactions to recruitment web sites: Visual and verbal attention, attraction, and intentions to pursue employment. Journal of Business Psychology, 28(3), p263-285, 2013. http://dx.doi.org/10.1007/s10869-012-9281-6.

[31] J. Simola, J. Kuisma, A. Oorni, L. Uusitalo, and J. Hyona, The impact of salient advertisements on reading and attention. Journal of Experimental Psychology: Applied, 17(2), p174-190, 2011. http://dx.doi.org/10.1037/a0024042.

[32] E. Higgins, M. Leinenger, and K. Rayner, Eye movements when viewing advertisements. Frontier in Psychology, 5, p1-15, 2010. http://dx.doi.org/10.3389/fpsyg.2014.00210.

[33] Z. Guan, and E. Cutrell, An eye-tracking study of the effect of target rank on web search. Paper presented at the SIGCHI Conference on Human Factors in Computing Systems, San Jose, CA, USA, April 30May 3, 2007. http://dx.doi.org/10.1145/1240624.1240691.

[34] J.H. Goldberg, M. Stimson, M. Kewenstein, N. Scott, and A.M. Wichansky, Eye-tracking in web search tasks: Design implications. Paper presented at the 2002 Symposium on Eye Tracking Research \& Applications, New Orleans, LA, USA, March 25-27, 2002. http://dx.doi.org/10.1145/507072.507082.

[35] B.D. Till, and D.W. Baack, Recall and persuasion: Does creative advertising matter? Journal of Advertising, 34(3), p47-57, 2005. http://dx.doi.org/10.1080/00913367.2005.10639201.

[36] R.P. Bagozzi, and A.J. Silk, Recall, Recognition, and the measurements of memory for print advertisements. Marketing Science, 2(2), p95-134, 1983. http://dx.doi.org/10.1287/mksc.2.2.95.

[37] S.N. Singh, M.L. Rothschild, and G.A. Churchill, Recognition versus recall as measures of television commercial forgetting. Journal of Marketing Research, 25(1), p72-80, 1988. http://dx.doi.org/10.2307/3172926.

[38] comScore.com, 19.2 million Spanish internet users watched 2.9 billion online videos in October 2010. Retrieved on December 11, 2010, from http://www.comscore.com/Insights/Press-Releases/2010/12/19.2-Millio n-Spanish-Internet-Users-watched-2.9-Billion-Online-Videos-in-Octob er-2010.

[39] comScore.com, UK engagement with online video descends with age. Retrieved on June 8, 2012, from http://www.comscore.com/Insights/Data-Mine/UK-Engagement-with- 
Online-Video-Descends-with-Age.

[40] comScore.com, comScore releases August 2014 U.S. online video rankings. Retrieved on September 17, 2014, from https://www.comscore.com/Insights/Market-Rankings/comScore-Relea ses-August-2014-US-Online-Video-Rankings.

[41] NECTEC (National Electronics and Computer Technology Center). Profiles of Internet users in Thailand. Bangkok: NECTEC press, 2012.

[42] PewResearch, Teens fact sheet. Retrieved on September 17, 2012, from http://www.pewinternet.org/fact-sheets/teens-fact-sheet/.

[43] S. Muylle, R. Moenaert, and M. Despontin, The conceptualization and empirical validation of web site user satisfaction. Information \& Management, 41(5), p543-560, 2004. http://dx.doi.org/10.1016/S0378-7206(03)00089-2.

[44] M. Pagendarm, and H. Schaumberg, Why are users banner-blind? The impact of navigational style on the perception of web banners. Journal of Digital Information, 2(1), p1-6, 2001.

[45] M. Koster, M. Ruth, K.-C. Hamborg, and K. Kaspar, Effects of personalized banner ads on visual attention and recognition memory. Applied Cognitive Psychology, 29(2), 181-192, 2014. http://dx.doi.org/10.1002/acp.3080

[46] N. Razzouk, and V.A. Seitz, Banner advertising and consumer recall. Journal of Promotion Management, 9(1-2), p71-80, 2003. http://dx.doi.org/10.1300/J057v09n01_07.

[47] Y.-C. Hsieh, K.-H. Chen, and M.-Y. Ma, Retain viewer's attention on banner ad by manipulating information type of the content. Computers in Human Behavior, 28(5), p1692-1699, 2012. http://dx.doi.org/10.1016/j.chb.2012.04.008.

[48] C.E. Tucker. The reach and persuasiveness of viral video ads. Marketing Science, 34(2), p281-296, 2015. http://dx.doi.org/10.1287/mksc.2014.0874. 
